\title{
STRUCTURAL EFFECTS OF VERAPAMIL ON CELL MEMBRANES AND MOLECULAR MODELS
}

\author{
MARIO SUWALSKY I, , MAURICIO MUÑOZI, SIGRID MENNICKENT ${ }^{2}$, CARLOS P. SOTOMAYOR ${ }^{3}$, \\ SILVIA BOLOGNIN ${ }^{4}$, PAOLO ZATTA
}

\author{
${ }^{1}$ Faculty of Chemical Sciences, University of Concepción, Casilla 160-C, Concepción, Chile \\ ${ }^{2}$ Faculty of Pharmacy, University of Concepción, Casilla 160-C, Concepción, Chile \\ ${ }^{3}$ Instituto de Química, Universidad Católica de Valparaíso, Valparaíso, Chile \\ ${ }^{4}$ CNR-Centre of Metalloproteins, University of Padova, Viale Colombo 3, Padova, Italy
}

(Received: November 6, 2009 - Accepted: December 28, 2009)

\begin{abstract}
Verapamil is one of the frequently prescribed calcium channel blockers used in the treatment of hypertension and angina pectoris. Results of evaluations of the therapy have led to reports of toxic effects. This study presents several evidences that verapamil affects human cells. Scanning electron microscopy observations of intact human erythrocytes indicated that they underwent morphological alterations as increasing verapamil concentrations starting from $5 \mu \mathrm{M}$ changed their discoid normal shape, and finally to hemolysis. Fluorescence spectroscopy on isolated unsealed human erythrocyte membranes confirmed these outcomes. In fact, the assays showed that verapamil induced a significant increase of the anisotropy parameters and a moderate one of the generalized polarization, indicative of enhanced order at the acyl chain and polar head regions of the erythrocyte membrane lipid bilayer. X-ray diffraction experiments on dimyristoylphosphatidylcholine and dimyristoylphosphatidylethanolamine bilayers, classes of the major phospholipids present in both outer and inner sides of the erythrocyte membrane, respectively showed that verapamil perturbed the polar head and acyl chain regions of both lipid bilayers. These interactions were found to be stronger with DMPC bilayers. On the other hand, human SH-SY5Y neuroblastoma cells incubated with verapamil suffered a sharp decrease of cell viability.
\end{abstract}

\section{INTRODUCTION}

Verapamil hydrochloride (Fig. 1) is a phenylalkylamine-derivate calcium-channel blocking agent. Chemically, it is a basic $(\log K=9.1)$ and highly hydrophobic compound $\left(\log \mathrm{P}_{o / \mathrm{w}}=9.1\right)^{1}$. It is used in the treatment of hypertension and angina pectoris, and has also been shown to be effective in the treatment of tachyarrhythmia, variant angina, and cardiomyopathy ${ }^{1-2}$. While verapamil is one of the frequently prescribed calcium channel blockers, certain results of the therapy evaluations have led to reports of toxic effects and overdoses at concentrations above $1000 \mathrm{ng} / \mathrm{ml}$. The exact molecular mechanism whereby verapamil works is still unknown; however, it has been suggested that it would be able to alter the potential distribution across biological membranes ${ }^{3}$. The effects of verapamil on cell membranes, particularly on those of human erythrocytes, has had limited coverage while most of the in vitro toxicological literature has focused on the drug effects on the heart ${ }^{4}$.

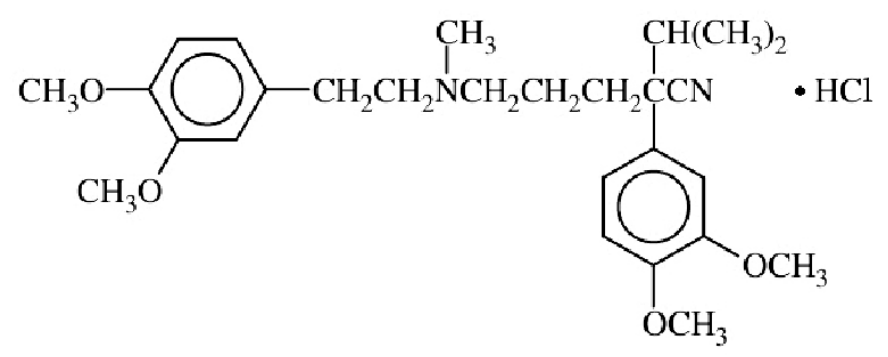

Fig. 1. Structural formula of verapamil hydrochloride.

The cell membrane is a diffusion barrier which protects the cell interior. Therefore, its structure and functions are susceptible to alterations as a consequence of interactions with chemical species. Intended to better understand the molecular mechanisms of the interaction of verapamil with cell membranes we have utilized human erythrocytes and molecular models of cell membranes. Human erythrocytes were chosen because having only one membrane and no internal organelles, is an ideal cell system for studying basic drug-biomembrane interactions ${ }^{5}$. On the other hand, although less specialized than many other cell membranes they carry on enough functions in common with them such as active and passive transport, as well as the production of ionic and electric gradients, to be considered representative of the plasma membrane in general. Molecular models of cell membranes consisted of dimyristoylphosphatidylcholine (DMPC) and dimyristoylphosphatidylethanolamine (DMPE) bilayers, representative of phospholipid classes located in the outer and inner monolayers of cell membranes, particularly of the human erythrocyte, respectively ${ }^{6-7}$. The capacity of verapamil to perturb the bilayer structures of DMPC and DMPE was assessed by X-ray diffraction, intact human erythrocytes were observed by scanning electron microscopy and isolated unsealed human erythrocyte membranes (IUM) were studied by fluorescence spectroscopy. These systems and techniques have been used in our laboratories to determine the interaction with and the membrane-perturbing effects of other drugs ${ }^{8-10}$ Additional experiments were performed in human neuroblastoma cells $\mathrm{SH}-$ SY5Y in order to test the toxicity after treatments with verapamil. The MTT (3-[4,5-dimethylthiazol-2-yl]-2,5-diphenyl tetrazolium bromide) assay was used to explore both the cell proliferation rate and cell viability. In the reaction, reductases of metabolically active, viable cells reduce the soluble MTT to the water-insoluble MTT-formazan which is spectrophotometrically detected.

\section{MATERIALS AND METHODS}

\section{X-ray diffraction studies of DMPC and DMPE multilayers}

Verapamil capacity to perturb the structures of DMPC and DMPE multilayers was evaluated by X-ray diffraction. Synthetic DMPC (lot $80 \mathrm{H}-$ 8371, MW 677.9) and DMPE (lot 084K-1676, MW 635.9) from Sigma (St. Louis, MO), and verapamil.HCl (MW 491.1) from Aldrich (Milwaukee, WI) were used without further purification. About $2 \mathrm{mg}$ of each phospholipid was introduced into $1.6 \mathrm{~mm}$ diameter special glass capillaries (Glas-Technik \& Konstruktion, Berlin, Germany, which were then filled with $150 \mu \mathrm{l}$ of (a) distilled water and (b) aqueous solutions in a range of concentrations. The specimens were X-ray diffracted after $30 \mathrm{~min}$ incubation at $30^{\circ} \mathrm{C}$ and $60^{\circ} \mathrm{C}$ with DMPC and DMPE, respectively. Specimen-to-film distances were 8 and $14 \mathrm{~cm}$, standardized by sprinkling calcite powder on the capillary surface. $\mathrm{Ni}$ filtered $\mathrm{CuKa}$ radiation from a Bruker Kristalloflex 760 (Karlsruhe, Germany) $\mathrm{X}$-ray generator was used. The relative reflection intensities were obtained in an MBraun PSD-50M linear position-sensitive detector system (Garching, Germany); no correction factors were applied. Experiments were performed at $18^{\circ} \mathrm{C} \pm 1{ }^{\circ} \mathrm{C}$, which is below the main phase transition temperature of both DMPC and DMPE. Higher temperatures would have induced transitions to more fluid phases making the detection of structural changes harder. Each experiment was performed in triplicate and in case of doubts additional experiments were carried out. 
Scanning electron microscope (SEM) studies of intact human erythrocytes

A range of verapamil concentrations was prepared in order to interact in vitro with red blood cells by incubating erythrocyte suspensions obtained from a human healthy donor not receiving any pharmacological treatment. Blood was obtained by puncture of the ear lobule; $100 \mu \mathrm{L}$ were received in an eppendorff tube containing $10 \mathrm{~mL}$ of heparin $(5000 \mathrm{UI} / 5 \mathrm{ml}) .900 \mathrm{~mL}$ of saline solution $(\mathrm{NaCl} 0.9 \%, \mathrm{pH} 7.4)$ was added. The tube was centrifuged (1000 rpm $\mathrm{x} 10 \mathrm{~min}$ ), the supernatant was discarded and replaced by the same volume of saline solution; the whole process was repeated three times. Fractions of this stock of red blood cells suspension (RBCS) in saline solution were placed in each one of five eppendorff tubes to prepare (A) the control, by mixing 150 $\mu \mathrm{L}$ saline solution plus $100 \mathrm{~mL}$ RBCS, and (B) a range of concentrations by mixing $100 \mu \mathrm{L}$ of RBCS in saline with $150 \mu \mathrm{L}$ of each verapamil concentration. All the samples were then incubated in an oven at $37^{\circ} \mathrm{C}$ for $1 \mathrm{~h}$, period in line with the larger effects of studied compounds on red cell shape ${ }^{11-12}$. Afterwards, they were fixed for $12 \mathrm{~h}$ at $4^{\circ} \mathrm{C}$ with $900 \mu \mathrm{L} 2.5 \%$ glutaraldehyde in distilled water, reaching a final fixation concentration of about $2.4 \%$. Finally, previously centrifuged samples at $1000 \mathrm{rpm}$ were washed in distilled, re-suspended in 200 $\mu \mathrm{L}$ of distilled water; drops of each one were placed on $\mathrm{Al}$ glass cover stubs, air-dried at room temperature, gold coated and examined in a scanning electron microscope (JEOL JSM-6380LV, Japan).

Fluorescence measurements of isolated unsealed human erythrocyte membranes (IUM).

Another approach used to analyze the influence of verapamil on the physical properties of human erythrocyte membranes was the measurement of two fluorescence parameters: anisotropy (r) and generalized polarization (GP) by fluorescence spectroscopy. This issue was achieved by using two different fluorescent probes: 1,6-diphenyl-1,3,5-hexatriene (DPH) and 6-dodecanoyl-2dimethylaminonaphthalene (laurdan). Isolated unsealed human erythrocytes membranes (IUM) were prepared with human blood obtained from healthy male donors according to the method of Dodge et al. ${ }^{13}$. Briefly, by means of both isotonic and hypotonic phosphate buffered saline (PBS), erythrocytes were separated from plasma, lysed and then washed in order to obtain the unsealed membranes, which were stored in isotonic solution at $-20^{\circ} \mathrm{C}$. IUM were incubated with DPH and Laurdan by addition of smalls aliquots of concentrated solution of the probe in tetrahydrofurane and ethanol, respectively, at $37^{\circ} \mathrm{C}$ for about one hour. Fluorescence spectra and anisotropy measurements were performed on a K2 steady-state and time-resolved spectrofluorometer (ISS Inc., Champaign, IL, USA) interfaced to computer, using the corresponding ISS software. Temperature was monitored before and after each measurement by a digital thermometer (Omega Engineering Inc., Stanford, CT, USA). Samples were measured using $5 \mathrm{~mm}$ path-length square quartz cuvettes. Anisotropy measurements were made in the $\mathrm{L}$ configuration using Glan Thompson prism polarizers in both exciting and emitting beams. Using an excitation wavelength of $360 \mathrm{~nm}$, the emission was measured with a WG-420 Schott high-pass filter (Schott, Mainz, Germany) with negligible fluorescence. DPH fluorescence anisotropy (r) was calculated according to the definition $r=\left(I_{\Perp}-I_{\perp}\right) /\left(I_{\|}-2 I_{\perp}\right)$, where $I_{\|}$and $I_{\perp}$ are the corresponding parallel and perpendicular emission fluorescence intensities with respect to the vertically polarized excitation light ${ }^{14}$. Laurdan fluorescence spectral shifts were quantitatively evaluated using the generalized polarization (GP) concept ${ }^{15}$, which is defined by the expression $\mathrm{GP}=\left(\mathrm{I}_{\mathrm{b}}-\mathrm{I}_{\mathrm{r}}\right) /\left(\mathrm{I}_{\mathrm{b}}+\mathrm{I}_{\mathrm{r}}\right)$, where $\mathrm{I}_{\mathrm{b}}$ and $\mathrm{I}_{\mathrm{r}}$ are the emission intensities at the blue and red edges of the emission spectrum, respectively. With excitation at 360 $\mathrm{nm}$ these intensities have been measured at the emission wavelengths of 440 and $490 \mathrm{~nm}$, which correspond to the emission maxima of laurdan in the gel and liquid crystalline phases, respectively ${ }^{16}$. Verapamil was incorporated in IUM suspensions by addition of adequate aliquots in order to obtain different concentrations in the $0-1 \mathrm{mM}$ range. These samples were then incubated for $10-15$ minutes at $37^{\circ} \mathrm{C}$. Blanks were prepared using samples without probes. Data presented in Table 1 represent mean values and standard error of 15 measurements in two independent samples.

\section{Viability studies on human neuroblastoma cells SH-SY5Y}

SH-SY5Y human neuroblastoma cells were purchased from ECACC (European Collection of Cell Culture, Salisbury, UK). SH-SY5Y with a cell cycle of $23 \mathrm{~h}$ were cultured in DMEM/ F12 (Gibco, Carlsbad, CA USA) medium containing $15 \%(\mathrm{v} / \mathrm{v})$ fetal bovine serum (FBS, Sigma Aldrich, St. Louis, MO), 100 units $/ \mathrm{ml}$ penicillin (Gibco, Carlsbad, CA USA) and streptomycin (100 mg/ml; Gibco, Carlsbad, CA USA), at $37^{\circ} \mathrm{C}$ with $5 \% \mathrm{CO}_{2}$ in a humidified atmosphere ( $90 \%$ humidity). The medium was replaced every 2 days. $0.25 \%$ Trypsin-EDTA solution and phosphate buffered saline (PBS) were obtained from Sigma Aldrich (St. Louis, MO). Cell viability was assessed using the 3-(4,5-dimethylthiazol-2-yl)-2,5-diphenyltetrazolium bromide (MTT) reduction assay (Sigma Aldrich St. Louis, MO). Briefly, SHSY5Y cells were seeded into 24 -well plates (at a density of $10 \times 10^{4}$ cells per well, in 1.5 $\mathrm{ml}$ medium). $2 \%$ FBS medium containing verapamil at a concentration range from $1 \mathrm{nM}$ to $0.5 \mathrm{mM}$ was added to the cells for $1,6,24$ and $48 \mathrm{~h}$. The drug was prepared as a $43 \mathrm{mM}$ stock solution in bi-distilled water. MTT $(5 \mathrm{mg} /$ $\mathrm{ml}$ ) was added to each well and incubated in the dark at $37^{\circ} \mathrm{C}$ for $3 \mathrm{~h}$ followed by cells lysis and spectrophotometric measurement at $550 \mathrm{~nm}$ (Microplate SPECTRAmax ${ }^{R}$ at 550). The MTT solution was carefully decanted off, and formazan was extracted from the cells with $1 \mathrm{ml}$ of acidic isopropanol $(0.04$ $\mathrm{M} \mathrm{HCl}$ in absolute isopropanol) in each well ${ }^{17}$. Colour was measured with a 96-well ELISA plate reader. All MTT assays were performed three times in duplicate. All readings were compared with the control, which represented $100 \%$ viability. Data regarding MTT assay were performed using T-test and Primer software, and values were reported as highly statistically significant if $P<0.01$ and statistically significant if $P<0.05$. Results are presented as mean \pm standard deviation.

\section{RESULTS}

\section{X-ray diffraction studies of DMPC and DMPE multilayers}

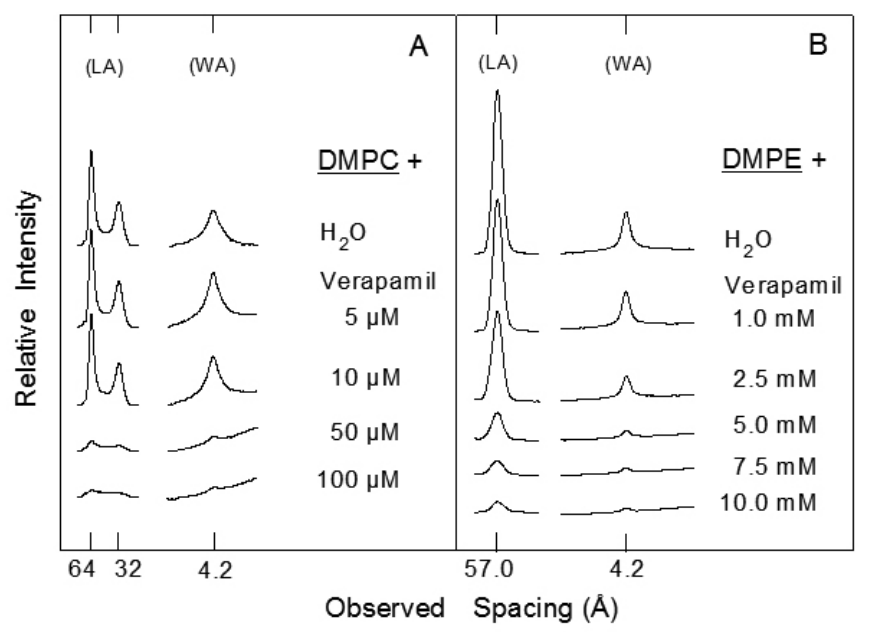

Fig. 2. Microdensitograms from X-ray diffraction patterns of (A) dimyristoylphosphatidylcholine (DMPC) and (B) dimyristoylphosphatidylethanolamine (DMPE) multibilayers in water and aqueous solutions of verapamil hydrochloride; (LA) and (WA) correspond to low- and wide-angle reflections, respectively.

Fig. 2A exhibits the results obtained by incubating DMPC with water and verapamil. As expected, water altered the structure of DMPC, as its bilayer repeat (phospholipid bilayer width plus the layer of water) increased from about $55 \AA$ in its dry crystalline form ${ }^{18}$ to $64 \AA$ when immersed in water, and its low-angle reflections (indicated as LA), which correspond to DMPC polar terminal groups, were reduced to only the first two orders of the bilayer repeat. On the other hand, only one strong reflection of $4.2 \AA$ showed up in the wide-angle region (indicated as WA), which corresponds to the average distance between fully extended acyl chains organized with rotational disorder in hexagonal packing. These results were indicative of the gel state reached by DMPC bilayers. Fig. 2A discloses that after exposure to $50 \mu \mathrm{M}$ verapamil, there was a considerable decrease of DMPC reflection intensities, which became almost negligible with $100 \mu \mathrm{M}$, an effect observed in both the low- and wideangle DMPC reflections. From these results, it can be concluded that verapamil produced a significant structural perturbation, affecting both the polar head and acyl chain regions of DMPC bilayers and, as a consequence, a disruption of the in-plane structure and the bilayer stacking. Results from similar experiments with DMPE are presented in Fig. 2B. As reported elsewhere, water did not significantly affect the bilayer structure of DMPE ${ }^{18}$. Thus, it can be observed that increasing verapamil concentrations reduced DMPE reflection intensities; however, these changes were attained with concentrations several orders of 
magnitude higher than those of DMPC.

Scanning electron microscope (SEM) studies of intact human erythrocytes

SEM examinations of human erythrocytes incubated with verapamil indicated that the drug induced different changes to the normal biconcave morphology of the red blood cells (Fig. 3A). Fig. 3B shows that when incubated with $5 \mu \mathrm{M}$ most of the cells presented stomatocytosis, an altered condition in which the erythrocytes show a cup-shaped form with evagination of one surface and a deep invagination of the opposite; Fig. 3C shows that when treated with $500 \mu \mathrm{M}$ the majority of the cells are stomatocytes, there are several knizocytes (red blood cells with two or three concavities due to indentations in the cell membrane), spherostomatocytes (cells with a visible change towards spheroid morphology with lightly or minor cupped profiles), and echinocytes (the erythrocytes show a spiny configuration, exhibiting blebs or protuberances in their surfaces); with $1.0 \mathrm{mM}$ the majority of the cells are knizocytes (Fig. 3D); $2.5 \mathrm{mM}$ induced the formation of spherocytes and knizocytes (Fig. 3E), while $5 \mathrm{mM}$ verapamil, the highest assayed concentraction, produced the lysis of most of the cells (Fig. 3F).
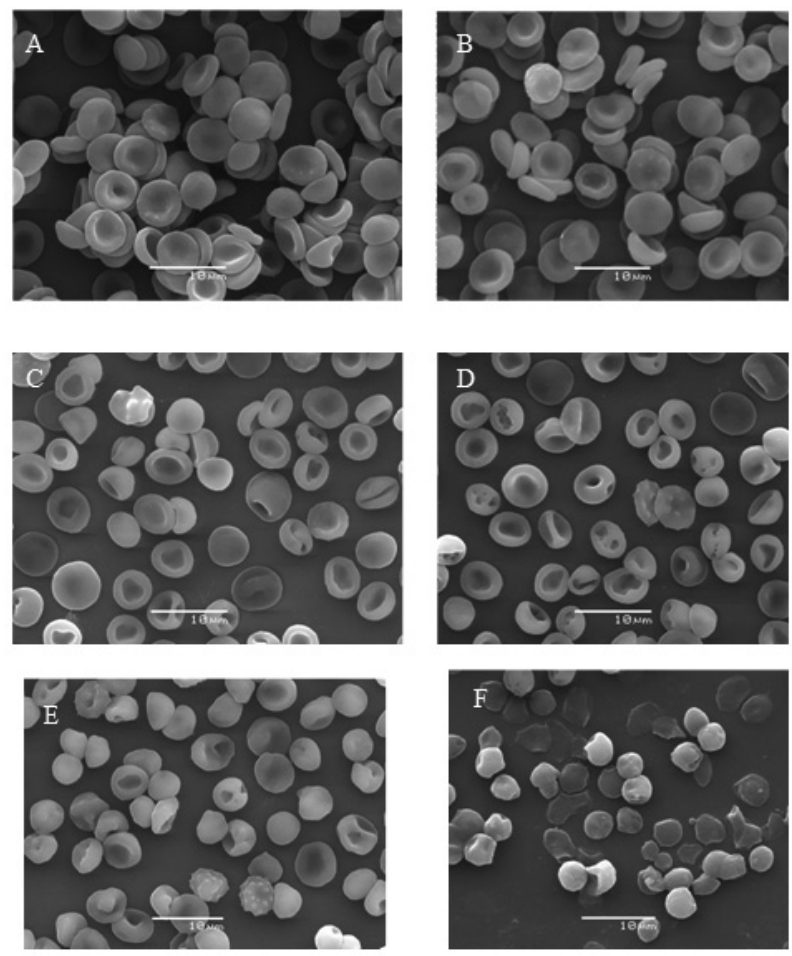

Fig. 3. Effect of verapamil on the morphology of human erythrocytes. Images obtained by scanning electron microscopy (SEM) of (A) control; (B) 5 $\mu \mathrm{M}$; (C) $0.5 \mathrm{mM}$; (D) $1 \mathrm{mM}$; (E) $2.5 \mathrm{mM}$; (F) $5 \mathrm{mM}$ verapamil hydrochloride.

Fluorescence measurements of isolated unsealed human erythrocyte membranes (IUM).

Verapamil concentration-dependent effects on IUM were explored at two different depths of the lipid bilayer: at the hydrophilic/hydrophobic interface level, estimated from the laurdan fluorescence spectral shift through the generalized polarization (GP) parameter, and in the deep hydrophobic core, determined by the DPH steady-state fluorescence anisotropy (r). Table 1 shows that the incorporation of $1 \mu \mathrm{M}$ and $10 \mu \mathrm{M}$ verapamil to IUM induced a $22 \%$ and $26 \%$ increase, respectively of the anisotropy (r) at $37^{\circ} \mathrm{C}$, implying an ordering effect in the hydrocarbon chain region of the lipid bilayer in the liquid crystalline state. Table 1 also shows no effects in the general polarization (GP) parameter, implying that verapamil, in these concentrations did not affect IUM polar head groups. However, $1 \mathrm{mM}$ (data not shown) induced a $12 \%$ increase in the general polarization (GP) parameter, implying a polarity and or a molecular dynamics decrease, presumably due to a moderate ordering of
IUM polar head groups.

Table 1. Effect of verapamil on the anisotropy (r) of DPH and the generalized polarization (GP) of laurdan embedded in isolated unsealed human erythrocyte membrane (IUM) at $37^{\circ} \mathrm{C}$.

\begin{tabular}{|c|c|c|}
\hline Concentration $(\mu \mathrm{M})$ & $\mathrm{r}(\mathrm{DPH})$ & GP (laurdan) \\
\hline 0 & $0.167 \pm 0.014$ & $0.246 \pm 0.012$ \\
\hline 1 & $0.203 \pm 0.013$ & $0.246 \pm 0.011$ \\
\hline 10 & $0.210 \pm 0.018$ & $0.246 \pm 0.012$ \\
\hline
\end{tabular}

\section{Viability studies on human neuroblastoma cells SH-SY5Y}

The effects of verapamil on human SH-SY5Y neuroblastoma cells were assayed at a concentration range from $1 \mathrm{nM}$ to $0.5 \mathrm{mM}$. Cells were incubated for 1, 6, 24 and $48 \mathrm{~h}$. As it can be appreciated in Fig. 4, a highly statistically significant decrease of cell viability was observed with $0.5 \mathrm{mM}$ verapamil treatment. This toxic effect was immediate, since it was evident only after 1 $\mathrm{h}$, and it was not recovered because it remained after $48 \mathrm{~h}$. The other tested concentrations were not able to produce any significant effect after 1, 6, and $24 \mathrm{~h}$; only after $48 \mathrm{~h}$ a significant decrease of cell viability was observed with $0.1 \mathrm{mM}$ verapamil.

These findings clearly showed that from $100 \mu \mathrm{M}$ and $10 \mu \mathrm{M}$ lies the threshold between toxic and non toxic concentration of verapamil.
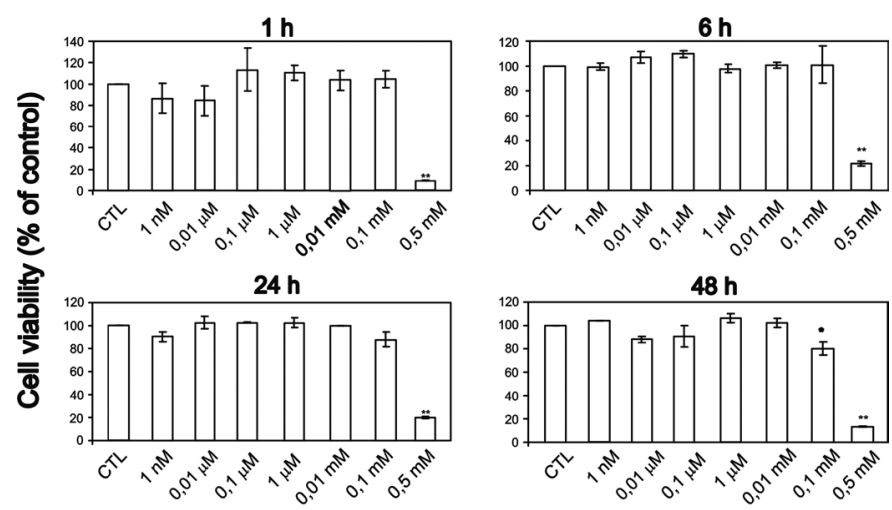

Fig. 4. Cytotoxicity assay in SH-SY5Y cells after treatment with verapamil hydrochloride in a range of concentrations. Neuroblastoma redox activity was measured by MTT assay. The data represented are mean $\pm \mathrm{SD}$ of two individual experiments, each done in duplicate. ${ }^{*} \mathrm{P}<0.05,{ }^{*} \mathrm{P}<0.01$ vs control.

\section{DISCUSSION}

The data herein reported demonstrates that verapamil affects human erythrocytes. Scanning electron microscopy observations of intact human erythrocytes indicated that they underwent morphological alterations as increasing verapamil concentrations from $5 \mu \mathrm{M}$ changed their discoid normal shape to stomatocytes, knizocytes, spherostomatocytes, and to hemolysis with $5 \mathrm{mM}$ verapamil. According to the bilayer couple hypothesis ${ }^{19-20}$, the morphological changes induced in erythrocytes by foreign molecules are due to the differential expansion of their two monolayers. Thus, speculated shapes (echinocytes) are induced when the added compound is inserted in the outer monolayer, whereas cup shapes (stomatocytes) arise when the compound accumulates in the inner monolayer. The X-ray diffraction experiments performed on bilayers made up of DMPC and DMPE, classes of the major phospholipids present in the outer and inner sides of the erythrocyte membrane, respectively showed that verapamil disordered the polar head and acyl chain regions of both DMPC and DMPE, these interactions being stronger with DMPC bilayers. DMPC and DMPE differ only in their terminal amino groups, these being ${ }^{+} \mathrm{N}\left(\mathrm{CH}_{3}\right)_{3}$ in DMPC and ${ }^{+} \mathrm{NH}_{3}$ in DMPE. Moreover, both molecular conformations are very similar in their dry crystalline phases ${ }_{18}$ with the hydrocarbon chains mostly parallel and extended, and the polar head groups lying perpendicularly to them. However, the gradual hydration of DMPC results in water filling the highly polar interbilayer spaces with the 
resulting increase of their width. This phenomenon allows the incorporation of verapamil into DMPC bilayers with the resulting disruption of its structure. Chemically verapamil hydrochloride is a highly hydrophobic compound (log $\mathrm{P}_{\text {in }}=9.1^{1}$; therefore, it is possible that intercalates into the membrane lipid bilayer locating into the vicinity of the polar/apolar membrane interface. On the other hand, DMPE molecules pack tighter than those of DMPC due to their smaller polar groups and higher effective charge, resulting in a very stable bilayer system that is not significantly affected by water. This organization did not prevent verapamil from interacting with DMPE and perturbing its structure. However, these effects were much milder than those observed in DMPC. Fluorescence spectroscopy on IUM at $37^{\circ} \mathrm{C}$ confirmed these results. In fact, the assays showed that verapamil induced a significant increase of DPH $r$ values, indicative of enhanced order at acyl chain regions of the erythrocyte membrane lipid bilayer. However, according to the bilayer-couple hypothesis the preferential interaction of verapamil with DMPC, a class of lipid mainly located in the outer monolayer of the erythrocyte membrane, should have induced echinocytosis instead of the observed stomatocytosis. The explanation for this discrepancy could be based on the lipid scrambling mechanism proposed by Schrier et al. ${ }^{21}$. According to it, some cationic amphipaths produce a rapid scrambling of the erythrocyte bilayer with phosphatidylcholines (PC) and sphyngomyelins (SM) moving inward while phosphatidylethanolamines (PE) moves outward along with phosphatidylserines (PS). Thus, the interaction of verapamil with $\mathrm{PC}$ in the inner monolayer would lead to stomatocytosis, an effect that can be produced by as little as $0.6 \%$ enrichment of the cytoplasmic monolayer ${ }^{21}$. However, interactions of verapamil with proteins located in the outer monolayer of the erythrocyte membrane cannot be disregarded. In fact, the ordering effect in the hydrocarbon chain region of the lipid bilayer detected in the fluorescence experiments occurred at very low drug concentrations with no subsequent significant effect. Therefore, it might be possible that the drug also interacted with the erythrocyte membrane proteins, with an ordering effect at the lipid protein interface.

Human SH-SY5Y neuroblastoma cells incubated with $0.5 \mathrm{mM}$ verapamil suffered a sharp decrease of cell viability. The primary pharmacological effect of verapamil is to block L-type $\mathrm{Ca}^{2+}$ channel. It has already been demonstrated that this drug, at massive concentrations, could alter $\mathrm{Na}^{+} / \mathrm{Ca}^{2+}$-exchanger transport activity ${ }^{22}$. This could partly explain the dramatic decrease of cellular viability at the highest assayed verapamil concentration $(0.5 \mathrm{mM})$.

Amazingly, the therapeutic range for plasma verapamil concentrations and the relationship of plasma concentration to clinical response and toxicity have not been clearly established. Toxic effects and overdoses at concentrations above $1000 \mathrm{ng} / \mathrm{ml}$ (equivalent to approx. $2 \mu \mathrm{M}$ verapamil) has been reported in serum ${ }^{1}$. A typical I.V. dose during cardiac care would be approximately 5-10 $\mathrm{mg}$ of verapamil hydrochloride ${ }^{23}$, which would be equivalent to a circulating dose of about $4 \mu \mathrm{M}$; however, its oral use in angina involve doses of 120-160 mg 2-3 times daily ${ }^{23}$; overdosing on just 10 tablets would result in circulating concentration of around $325 \mu \mathrm{M}{ }^{24}$. Our experimental results showed that 5 $\mu \mathrm{M}$ verapamil altered the shape of erythrocytes inducing stomatocytosis, 1 $\mu \mathrm{M}$ affected the anisotropy of DPH in IUM, and $50 \mu \mathrm{M}$ almost completely perturbed the bilayer structure of DMPC. As reported by Watts and Handy ${ }^{24}$ $50 \mu \mathrm{M}$ and higher verapamil concentrations caused hemolysis in vitro in a time dependent manner; although they could not identify the precise mechanism of this effect, they suggested a drug action on several ion-transport pathways and non-specific oxidative membrane damage.

Our experimental findings are certainly of interest as they demonstrated that verapamil interacts with the human erythrocyte membrane affecting the cell morphology. It must be considered that alteration of the normal biconcave shape of red blood cells increases their resistance to entry into capillaries, which could contribute to a decreased blood flow, loss of oxygen, and tissue damage through microvascular occlusion ${ }^{25-26}$. Functions of ion channels, receptors and enzymes immersed in cell membrane lipid moieties also might be affected. Accordingly to Lee ${ }^{27}$, integral membrane proteins are not rigid entities around which the lipid bilayer distorts enough as to provide the strongest interactions. Rather, both the lipid and proteins will distort to provide the best interactions, with the result that protein function will be affected by the structure of the surrounding lipid bilayer. These findings may also provide a new insight into the possible mechanism of action of verapamil.

\section{ACKNOWLEDGEMENTS}

Authors thank Fernando Neira for his technical assistance. This work was supported by FONDECYT (grant 1090041).

\section{REFERENCES}

1. M. Rambla-Alegre, M.T. Gil-Agustí, M.E. Capella-Peiró, S. Carda-Broch, J.S. Esteve-Romero, J. Chromatogr. B, 839, 89, (2006).

2. Y. Ozkul, Clin. Neurophysiol. 118, 2005, (2007).

3. E.E. Pohl, A.V. Krylov, M. Block, P. Pohl, Biochim. Biophys. Acta 1373, 170, (1998).

4. R. Gistri, F. Cecchi, L. Choudhury, A. Montereggi, O. Sorace, P.A. Salvadori, P.G. Camici, Am. J. Cardiol. 74, 363, (1994).

5. J.Y. Chen, W.H. Huestis, Biochim. Biophys. Acta 1323, 299, (1997).

6. J.M. Boon, B.D. Smith, Med. Res. Rev. 22, 251, (2000).

7. P.F. Devaux, A. Zachowsky, Chem. Phys. Lipids 73, 107, (1994).

8. M. Suwalsky, F. Villena, C.P. Sotomayor, S. Bolognin, P. Zatta, Biophys. Chem. 135, 7, (2008).

9. M. Suwalsky, S. Mennickent, B. Norris, F. Villena, C.P. Sotomayor, Toxicol. in Vitro 20, 1363 (2006).

10. M. Suwalsky, M. Manrique, F. Villena, C.P. Sotomayor, Biophys. Chem. 141, 34 (2009).

11. B. Zimmermann, D.M. Soumpasis, Cell Biophys. 7, 115, (1985).

12. S.V.P. Malheiros, M.A. Brito, D. Brites, M.N. Correa, Chemico-Biol. Interact. 126, 79, (2000).

13. J.T. Dodge, C. Mitchell, D.J. Hanahan, Arch. Biochem. Biopys. 100, 119, (1963).

14. J.R. Lakowicz, Principles of fluorescence spectroscopy, $2^{\text {nd }}$ edition, Kluwer/ Plenum, New York, 1999.

15. T. Parasassi, E. Gratton, J. Fluoresc. 5, 59, (1995).

16. T. Parasassi, G. De Stasio, A. d'Ubaldo, E. Gratton, Biophys. J. 57, 1179, (1990).

17. M.S. Shearman, S.R. Hawtin, V.J. Tailor, J. Neurochem. 65, 218, (1995).

18. M. Suwalsky, Phospholipid bilayers in Polymeric Materials Encyclopedia, J.C. Salamone ed., vol. 7, CRC, Boca Raton, FL, 1996; pp. 5073-5078.

19. M.P. Sheetz, S.J. Singer, Proc. Natl. Acad. Sci. USA 71, 4457, (1974).

20. G. Lim, M. Wortis, R. Mukhopadhyay, Proc. Natl. Acad. Sci. USA 99, 16766, (2002).

21. S.L. Schrier, A. Zachowski, P.F. Devaux, Blood 79, 782, (1992).

22. M.M. Hosey, M. Lazdunsky, J. Membr. Biol. 104, 81, (1988).

23. ABPI, Association of the British Pharmaceutical Industry Data Sheet Compendium. Data Publications, London, 1988.

24. T.J. Watts, R.D. Handy, Toxicol. in Vitro 21, 835, (2007).

25. S.L. Winski, D.E. Carter, J. Toxicol. Environ. Health A, 53, 345, (1998).

26. S. Svetina, D. Kuzman, R.E. Waugh, P. Zibert, B. Zeks, Bioelectrochem. 62, 107, (2004).

27. A.G. Lee, Biochim. Biophys. Acta 1666, 62. (2004). 